\title{
COVID-19 and Mucormycosis of the Craniofacial skeleton: Causal, Contributory or Coincidental?
}

\author{
Dinesh Kumar Verma ${ }^{1} \cdot$ Rishi Kumar Bali ${ }^{2}$
}

Published online: 27 March 2021

(C) The Association of Oral and Maxillofacial Surgeons of India 2021

The outbreak of Corona virus disease 2019 (COVID-19) has changed the landscape of medicine in many ways. Being a new disease entity with a high infectivity rate and associated fatality, it is also associated with certain morbidities induced by novel therapeutic protocols resulting in additional challenges. One such issue, osteomyelitis of the craniofacial skeleton, has been a point of constant discussions in online, offline academic groups and print media. There were many anecdotal cases of fungal osteomyelitis of craniofacial skeleton in patients previously diagnosed with COVID-19 leading to a common inference that incidence of the osteomyelitis has increased drastically due to COVID-19. It has been almost a year since these reports started coming in, and a search for evidence would help in clearing the air.

Search of PubMed database with search terms (osteomyelitis of maxilla OR craniofacial osteomyelitis) AND (COVID-19 OR Corona virus OR SARS-CoV-2) surprisingly did not return results. Search with terms (mucormycosis OR zygomycosis OR fungal osteomyelitis) AND (COVID-19 OR Corona virus OR SARS-CoV-2) returned 16 results. Out of these only two reports had clinical features which pointed towards fungal maxillary osteomyelitis $[1,2]$. One reported premaxillary swelling in association

Rishi Kumar Bali

rshbali@hotmail.co.uk

Dinesh Kumar Verma

dineshverma@yahoo.com

1 Department of Dentistry, All India Institute of Medical Sciences, Bilaspur, Himachal Pradesh 174001, India

2 Department of Oral and Maxillofacial Surgery, DAV Dental College and MDM General Hospital, Yamunanagar, Haryana 135001, India with rhino-orbito-cerebral mucor [1] and the other reported maxillary hypoesthesia in association with rhino-orbital mucor [2]. All the other case reports were of rhinocerebral, rhino-orbital, rhino-orbito-cerebral, pulmonary, disseminated or gastrointestinal mucormycosis. There was a strong association between presence of history of COVID-19 and mucormycosis. However, presence of two important confounders, diabetes and other comorbid conditions, was also observed. Mucormycosis is usually seen in immunocompromised individuals with diabetes being an independent risk factor. Diabetes has also been identified as an independent variable associated with severity of COVID-19 infection and hospitalization. To add to this cauldron the current guidelines suggest use of steroids in admitted patients requiring oxygen support [3]. SARS-CoV-2 virus itself has been implicated in causing an impaired cell mediated immune response with drop in $\mathrm{CD} 4+\mathrm{T}$ and $\mathrm{CD} 8+\mathrm{T}$ cell counts [4]. A combination of these factors makes the hospitalized COVID-19 patients a high-risk group for fungal infections like aspergillosis and mucormycosis. Whereas aspergillosis is associated with fungus balls of the sinuses without tissue invasion, mucormycosis is an angioinvasive fungal infection which causes ischemic necrosis of the areas involved. The clinical course is rapid with case fatality rate of $46 \%$ [5]. Clinicians should have a high index of suspicion in immunocompromised patients and COVID-19 patients, with history of hospital admission for oxygen support, as this category of patients frequently has comorbid conditions like diabetes, renal failure and is usually administered steroids / antibiotics as part of COVID-19 management protocol.

Diagnosis of fungal infections is based on direct microscopy with $\mathrm{KOH}$ mounted slides (especially if urgent treatment is warranted) and confirmatory diagnosis is based on definitive histological evidence of tissue invasion and 
culture. Many a times the nature of the disease affords little time for fungal cultures (fungal cultures are costly and take up to 6 weeks). Treatment recommended ranges from removal of fungal ball in cases of aspergillosis to early aggressive surgical treatment in mucormycosis. In addition, systemic antifungal treatment with amphotericin B (lipid complex or liposomal), posaconazole oral suspension or Isavuconazonium (IV or oral) has to be instituted [6]. The sudden spurt in this devastating illness which has got immense financial implications has left the medical community befuddled. With conventional Amphotericin (the drug of choice) being significantly nephrotoxic, the safer Liposomal Amphotericin sets the patient back by Rs. 300,000-400,000-a financial burden our nascent public healthcare system can ill-afford.

There is paucity of reports of mucormycosis in nondiabetic COVID-19 patients who did not receive steroids and reports of COVID-19 immunocompetent patients who received steroids having developed mucormycosis of craniofacial skeleton. This makes it difficult to establish a causal effect relationship between COVID-19 and craniofacial mucormycosis. This forces us to focus on another matter-documentation and publication of case reports. Adequate documentation is necessary to generate evidence. Without evidence, even a significant event runs the risk of becoming hearsay. Publications in scientific literature will also help assimilate management experience and provide a reservoir of information for clinicians managing such patients, thus helping them render better care.

Is COVID-19 causal, contributory or coincidental? It is impossible to judge that yet as the current evidence does not suggest a direct causal effect relationship but the combination of influence of the virus on cell-mediated immunity, association of immunocompromised conditions and treatment protocols affecting immune mechanisms creates ideal conditions for invasive fungal infections. Multicenter audits to study the incidence and patterns of fungal infections of the craniofacial skeleton in both the healthy and comorbid patient cohorts might help in establishing or dispelling its proposed correlation with COVID19.

\section{References}

1. Mehta S, Pandey A (2020) Rhino-orbital mucormycosis associated with COVID-19. Cureus 12(9):e10726. https://doi.org/10.7759/ cureus.10726 (PMID: 33145132; PMCID: PMC7599039)

2. Waizel-Haiat S, Guerrero-Paz JA, Sanchez-Hurtado L, CallejaAlarcon S, Romero-Gutierrez L (2021) A case of fatal rhinoorbital mucormycosis associated with new onset diabetic ketoacidosis and COVID-19. Cureus 13(2):e13163. https://doi.org/10. 7759/cureus.13163 (PMID: 33575155; PMCID: PMC7870113)

3. https://www.covid19treatmentguidelines.nih.gov/therapeuticmanagement/

4. Gangneux JP, Bougnoux ME, Dannaoui E, Cornet M, Zahar JR (2020) Invasive fungal diseases during COVID-19: we should be prepared. J Mycol Med. 30:100971

5. Jeong W, Keighley C, Wolfe R, Lee WL, Slavin MA, Kong DCM, Chen SC (2019) The epidemiology and clinical manifestations of mucormycosis: a systematic review and meta-analysis of case reports. Clin Microbiol Infect 25(1):26-34. https://doi.org/10. 1016/j.cmi.2018.07.011 (Epub 2018 Jul 21 PMID: 30036666)

6. Cornely OA, Alastruey-Izquierdo A, Arenz D, Chen SCA, Dannaoui E, Hochhegger B et al (2019) Global guideline for the diagnosis and management of mucormycosis: an initiative of the European Confederation of Medical Mycology in cooperation with the Mycoses Study Group Education and Research Consortium. Lancet Infect Dis 19(12):e405-e421

Publisher's Note Springer Nature remains neutral with regard to jurisdictional claims in published maps and institutional affiliations. 